\title{
Days Between Birth and Diagnosis
}

National Cancer Institute

\section{Source}

National Cancer Institute. Days Between Birth and Diagnosis. NCI Thesaurus. Code C156418.

The time interval from a person's date of birth to the date of initial pathologic diagnosis, represented as a calculated number of days. 